\title{
Grey Prediction System Based on Browser/Server Structure
}

\author{
${ }^{*}$ Hui Peng ${ }^{1}$,Wenqi Qu ${ }^{1}$ \\ 'Education Technology Center, Beijing International Studies University. Beijing, China, 100024. \\ *email: penghui@bisu.edu.cn
}

Keyword: grey prediction, browser/server structure, decision support system

\begin{abstract}
Grey prediction algorithm has been used in prediction area extensively. A grey prediction system based on grey prediction algorithm and Browse/Server structure is proposed in this paper. In this system browser produces user interface, receives inputs and pass them to server. Server predicts results with the help of grey algorithm and database, then server return the results to browser. Browser shows results through table and graph. This system is working in the decision support system of department of transportation Hunan province and supplies decision support for the transportation.
\end{abstract}

\section{Introduction:}

Grey theory was proposed by the professor Julong Deng from Huazhong University of Science and Technology in 1982 to deal with the data in grey systems[1]. It analyses the system which includes insufficient information and half-baked data, analyses data relations and promotes models to predict or decide all information in the system. Its characteristic is dealing with discrete data with few samples and many inputs efficiently. Grey theory includes six methods: grey building, grey relating, grey predicting, grey modeling and grey controlling[1,2]. Among them, grey predicting applies consecutive differential equation to give a general analysis and a long time prediction for few discrete data. For the last decade, the grey prediction model was widely used in many fields and has produced excellent predict results.

When we construct grey system with grey theory, there are often three aspects we should consider :the storage of data, the execute of the algorithm and the user interface. Files or database is often the way of data storage. When a grey system run under web environment, the interface is often embedded in the web pages[3].

In the grey prediction system of the department of transportation Hunan province, we select Java Applet as the client program because it can be run in web page. We select Java Application as the server program. It runs as the application server to execute grey algorithm and communicate with client and database. The original data is saved in database. In this paper, the system structure was introduced in part2. the program was introduced in part 3.

\section{The Structure of Browser/Server System}

Figure 1 shows the browser server structure. There are two main parts In this structure. One is the client which runs a Java Applet embedded in web page of a browser. The client includes three important function: interface of inputs and outputs, protocol analysis and socket connection with application server. The other is the application server. It includes four parts: socket connection with client, protocol analysis, prediction algorithm and connection with database. The work flow of this system includes 6 steps:1. Application starts, connects with database and waits for the request from client. 2. Client send a request to Web server(such as 
IIS, Apache) and get web page which includes Java Applet. 3. Java applet runs to connect application server and produce interface.3.client sends inputs to prediction server4.server executes grey prediction algorithm with inputs and original data from database. 5. Server returns the results to client. 6. Client shows the results. The advantages of this structure lie in two aspects. One is the simple client environment. The client needs only a browser to run applet, no installation and maintenance of client software. The other advantage is the system utilize the calculating ability of the server sufficiently.

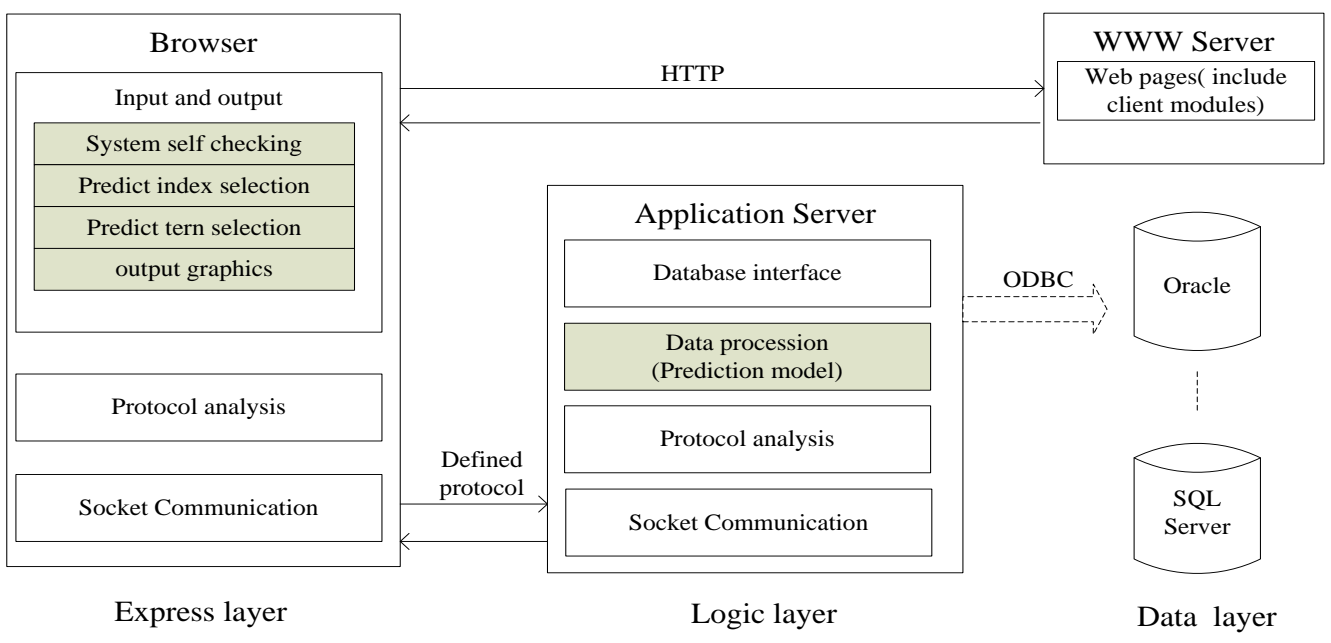

Figure 1: Browser/Server Structure of the Grey Prediction System

\section{Java Program of Prediction System}

In this part, we provide parts of program of this system. There are two java programs. One is the client program named predict.java. The other is the server program named predict_server[4]. The results of this system applying this system in traffic indexes prediction in Hunan province, more than fifty traffic indexes are predicted successfully[5].

\section{public class predict extends Applet}

$\{$ Socket client=null;

public void init()

\{ super.init();

NetworkConnection();

CreateImage();

CreateInterface();

\}

public void NetworkConnection()

$\{$ try

\{ String url=this.getCodeBase().toString();

url=url.substring $(7)$;

url=url.substring $(0$, url.indexOf('/') $)$;

client=new Socket(url,100);

in=new DataInputStream(client.getInputStream());

out=new DataOutputStream(client.getOutputStream());

out.writeBytes("Hello,My Server");

out.write(13); out.write(10); out.flush();

inLine $=$ in.readLine();

if (inLine.equalsIgnoreCase("Hello,My Client"))

System.out.println("TCP/IP Connection Created Successfully."); 


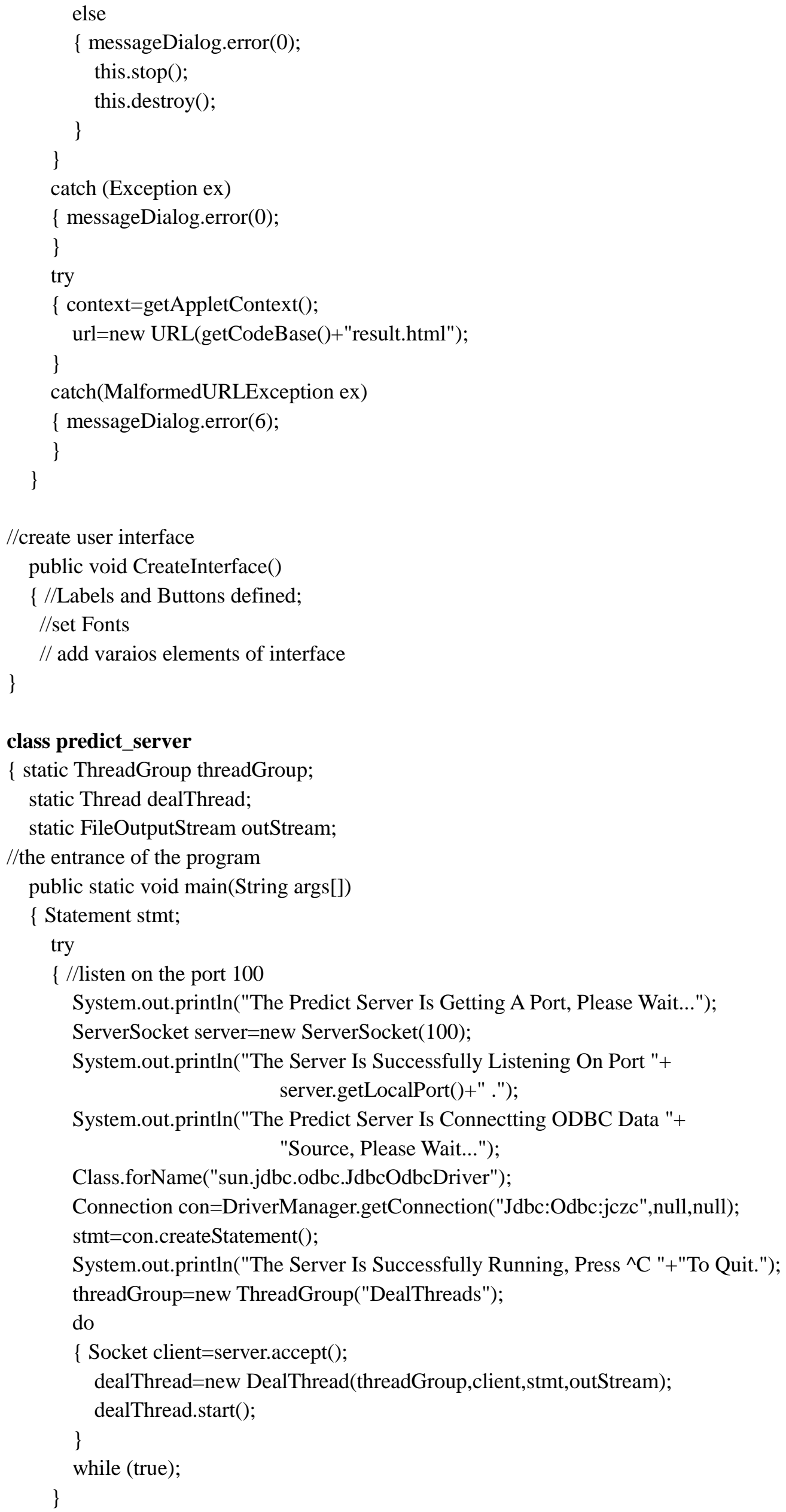




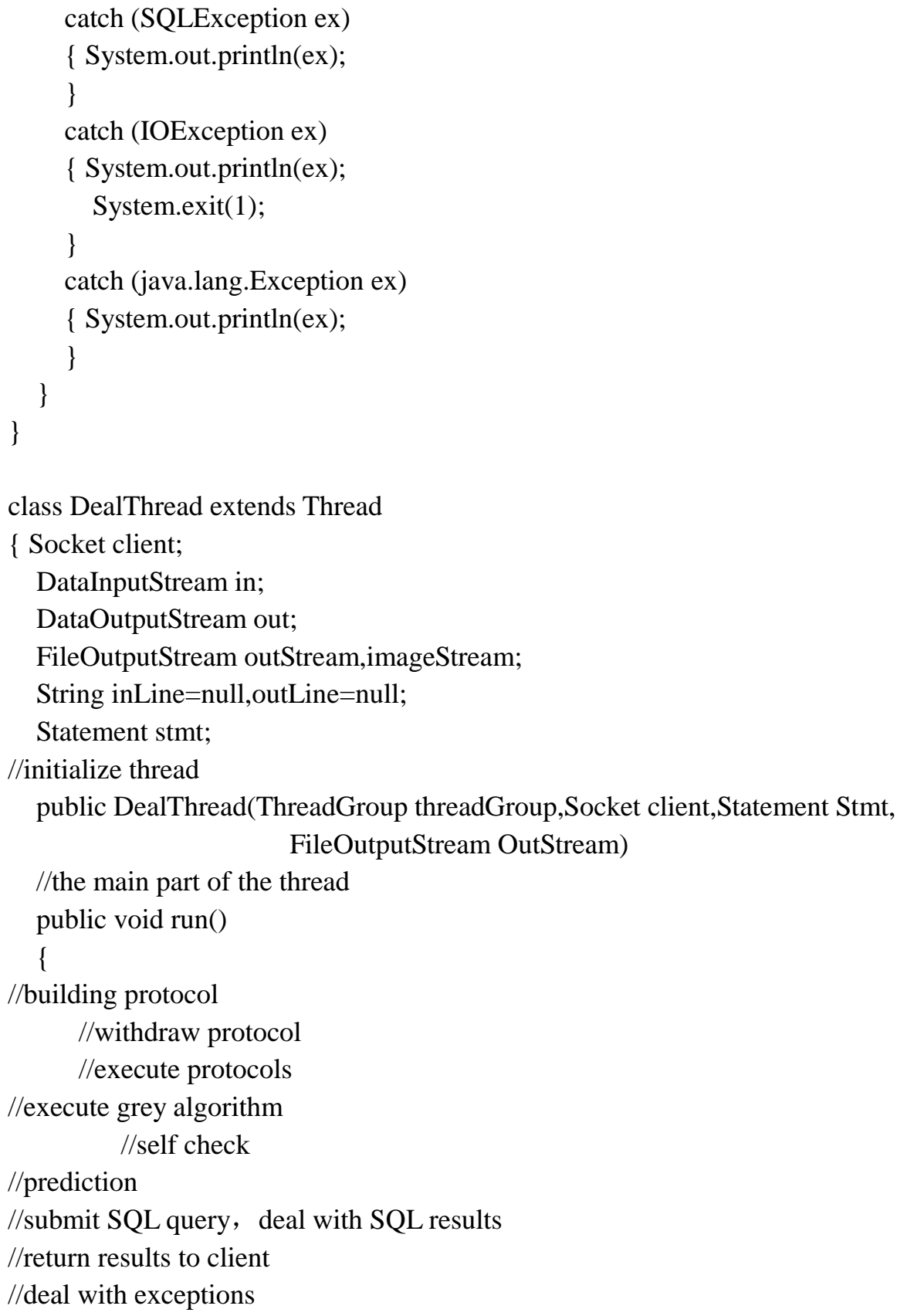

\section{References}

[1] Julong Deng. The Basic Method of Grey Theory. Huazhong University of Science and Technology Press. 1987(in Chinese)

[2] Julong Deng. The Grey Control System. H uazhong University of Science and Technology Press. 1993(in Chinese)

[3] Zhongzhi Shi. Knowledge discovery. Tsinghua press,2001(in Chinese)

[4] Jamie Jaworski. Java Developer's Guide. Sams.net Press 1996(in Chinese)

[5] Hui Peng, Wenqi Qu, Junjian Tang et al.. Traffic Indexes Prediction Based on Grey Prediction Model The $6^{\text {th }}$ International Symposium on Computational Intelligence and Design.2006, Hangzhou. 